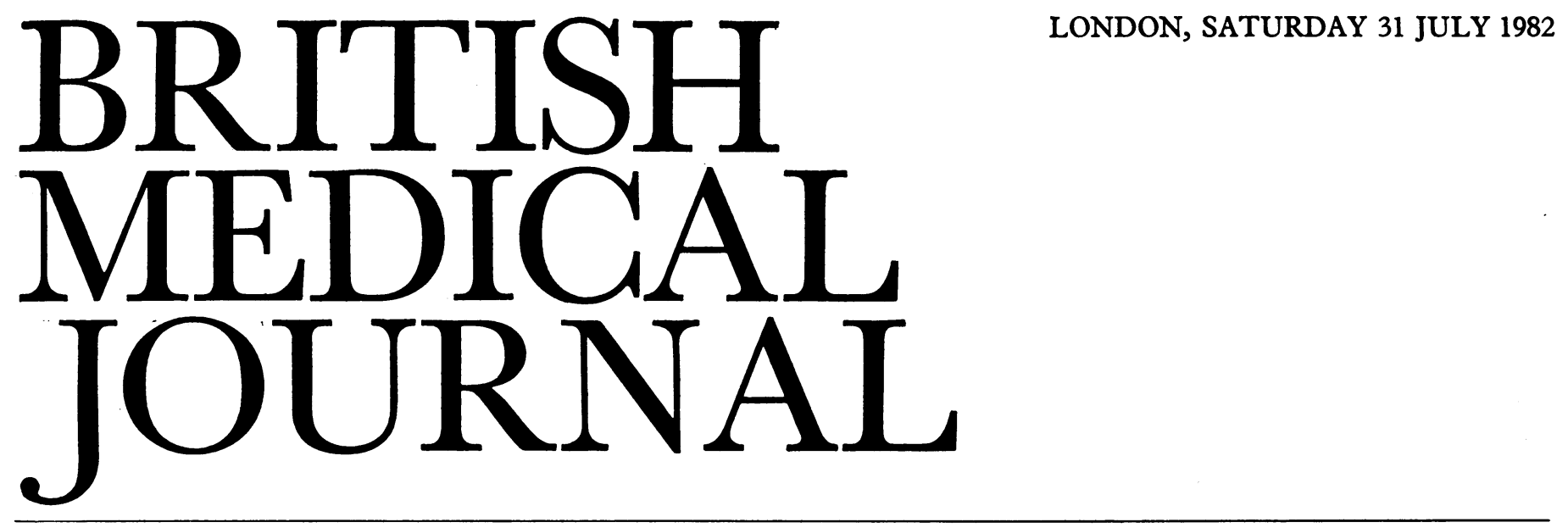

\title{
The diagnosis of sarcoidosis
}

No proper definition of sarcoidosis is possible while its aetiology remains unknown. In 1976 an international subcommittee" reported that: "Sarcoidosis is a multisystem granulomatous disorder of unknown etiology most commonly affecting young adults and presenting most frequently with bilateral hilar lymphadenopathy, pulmonary infiltration, and skin or eye lesions. The diagnosis is established most securely when clinico-radiographic findings are supported by histologic evidence of widespread non-caseating epithelioid cell granulomas in more than one organ or a positive Kveim-Siltzbach skin test." Among the causes that have been suggested for sarcoidosis are Mycobacterium tuberculosis, atypical mycobacteria, Epstein-Barr virus, fungi, and pine needles. Beryllium produces a sarcoid-type response. Mitchell and Scadding ${ }^{2}$ have reported evidence of a transmissible agent, but whether this represents an infection or an autoantigenic transmission remains to be proved.

Sarcoidosis occurs world wide, perhaps most commonly and severely in people of African origin; its true incidence is unknown but various surveys have reported rates of between one and 64 per 100000 population. ${ }^{4}$ Israel $^{5}$ considers that genetic factors are probably responsible for the difference in incidence of sarcoidosis. Though sarcoidosis normally resolves with only mild symptoms it can be both serious in vital organs and progressive and disabling, particularly in the lungs. Sarcoidosis presents most often in respiratory clinics but may present in almost any outpatient clinic.

Some clinicians accept the diagnosis without detailed investigation because sarcoidosis may be so typical in clinical presentation. For example, a young adult with slight malaise, chest discomfort, a cough, and bilateral hilar gland enlargement-especially if preceded by erythema nodosum and found to have a negative tuberculin test result-would be investigated further, by some clinicians, only if the subsequent course proved unfavourable. Other physicians prefer to establish some more objective diagnostic features in all cases, and all clinicians are faced with patients presenting problems of differential diagnosis in whom additional investigation is essential. Since there is no specific test for sarcoidosis the diagnosis must be made from a combination of clinical features, contributory investigations, and simultaneous exclusion of other diseases.

The most important single criterion is the finding of typical granulomas-histologically comprising nodular collections of epithelioid cells, a few multinucleated giant cells, and lymphocytes with no, or rarely little, necrosis, and no acid-fast bacilli. Clinically suspect tissue provides positive histological findings in $70-80 \%$ of cases. Naturally the ease of biopsy varies, but it is usually feasible; since the intrathoracic organs are most often affected biopsy of abnormal mucous membrane should be possible or a transbronchial lung biopsy may be done using a fibrescope. Unfortunately, the sarcoid reaction is not specific and may be seen in or near to various infective, chemical, and neoplastic lesions-though, in such cases, there are usually other pointers to the correct diagnosis. Calcium metabolism ${ }^{6}$ is abnormal in sarcoidosis, and hypercalciuria is common (though hypercalcaemia is unusual). These features are not diagnostic.

The Kveim test is widely used and a clearly positive result strongly indicates sarcoidosis; false-positive results are rare. The test relies on the fact that a saline suspension of sarcoid tissue introduced intradermally will induce a granuloma of sarcoid type when biopsied at four to six weeks. Unfortunately test preparations vary, and the Kveim test cannot be regarded as definitive.

In recent years much effort has been employed in the search for the cause or causes (sarcoidosis may be a common response to several inimical influences), for tests of diagnostic value, for degrees of activity, and for extent of the disease. The cause of sarcoidosis remains undiscovered, but three techniques of diagnostic value have been discovered. Gallium-67, a radioisotope with a half life of 74 hours, accumulates in rapidly dividing cells and has been shown to do so in active sarcoidosis. ${ }^{7}$ Such accumulation is not specific, since it also occurs in other infections and neoplasms, but the test can be used in repeat scanning to distinguish active from fibrotic disease, thus providing evidence of progress during treatment. ${ }^{8}$ Angiotensinconverting enzyme activity is usually raised in active sarcoidosis $^{9}$ : the source is not clear but it probably comes in part at least from the epithelioid cells of granulomatous lesions. Unfortunately the angiotensin-converting enzyme test is not specific; values may be raised in silicosis and they do not adequately reflect the intensity of early and potentially reversible lesions.

Bronchoalveolar lavage is performed by wedging a bronchofibrescope in a bronchus of the right middle lobe or lingular segment, passing $200-300 \mathrm{ml}$ sterile isotonic saline in $30 \mathrm{ml}$ 
aliquots into the lung, and aspirating after each aliquot. ${ }^{10}$ The lavage fluid is then analysed. $T$ lymphocytes appear to be especially important in mediating the lung's inflammatory response, and their numbers are a good guide to the intensity of the alveolitis. ${ }^{1112}$ The investigation can be repeated to assess progress.

These methods, gallium-67, angiotensin-converting enzyme activity, and bronchoalveolar lavage, are contributing to a better understanding of sarcoidosis and its extent and state of activity in individual patients. Some uncertainty persists about how the three methods correlate with each other, but perhaps gallium-67 and bronchoalveolar lavage do so best. ${ }^{811} 12$ Each of the methods will no doubt be refined; all require skill and equipment and are most appropriate in research at present.

\section{Graham W Poole}

Consultant Respiratory Physician,

Chest Clinic,

Hammersmith Hospital,

London $\mathrm{W} 12$ OHS

1 James DG, Turiaf J, Hosoda Y, et al. Description of sarcoidosis: report of the Subcommittee on Classification and Definition. Ann NY Acad Sci $1976 ; 278: 742$.

${ }^{2}$ Mitchell DN. The nature and physical characteristics of a transmissible agent from human sarcoid tissue. Ann NY Acad Sci 1976;278:233-48.

${ }^{3}$ Mitchell DN, Scadding JG. Sarcoidosis. Am Rev Respir Dis 1974;110: 774-802.

${ }^{4}$ Lofgren S, moderator. Epidemiology of sarcoidosis. Methodological aspects of mass chest radiography. Acta Med Scand 1964;suppl 425 92-177.

${ }^{5}$ Israel HL. Sarcoidosis. In: Loudon RG, ed. Basics of RD. Vol 7. New York: American Thoracic Society, 1978:1-6.

${ }^{6}$ Reiner M, Sigurdsson G, Nunziata V, Malik MA, Poole GW, Joplin GF. Abnormal calcium metabolism in normocalcaemic sarcoidosis. $\mathrm{Br} \mathrm{Med} \mathcal{f}$ 1976;ii:1473-6.

${ }^{7}$ McKusick KA, Soin JS, Ghiladi A, Wagner HN Jr. Gallium 67 accumulation in pulmonary sarcoidosis. $\mathcal{F} A M A 1973 ; 223: 688$.

8 Beaumont D, Harry JY, Sapene M, Bourguet P, Larzul JJ, De Labarthe B. Gallium-67 in the evaluation of sarcoidosis: correlations with serum angintensin-converting enzyme and bronchoalveolar lavage. Thorax $1982 ; 37: 11-8$.

- Lieberman J. Elevation of serum angiotensin-converting-enzyme (ACE) level in sarcoidosis. $A m \mathcal{F}$ Med 1975;59:365-72.

${ }^{10}$ Greening AP. Bronchoalveolar lavage. $\mathrm{Br} \mathrm{Med} \mathcal{F}$ 1982;284:1896-7.

${ }^{11}$ Rossman MD, Dauber JH, Cardillo ME, Daniele RP. Pulmonary sarcoidosis: correlation of serum angiotensin-converting enzyme with blood and bronchoalveolar lymphocytes. Am Rev Respir Dis 1982;125:366-9.

12 Schoenberger CI, Line BR, Keogh BA, Hunninghake GW, Crystal RG. Lung inflammation in sarcoidosis: comparison of serum angiotensinconverting enzyme levels with bronchoalveolar lavage and gallium-67 scanning assessment of the $T$ lymphocyte alveolitis. Thorax $1982 ; 37$ : $19-25$.

\section{Morning-after pills}

Postcoital contraception has been in use in many countries for more than a decade, ${ }^{12}$ but some doctors still shrink from intervention in the reproductive process after ovulation-even when the timing is clearly before implantation. Around $80 \%$ of women who need postcoital treatment opt for hormone pills. $^{3}$ The others have an intrauterine contraceptive device fitted. The relative merits of the two methods were discussed earlier this year at a symposium organised by the Pregnancy Advisory Service.

To be effective, hormonal treatment should be started within 72 hours of unprotected intercourse; thereafter the failure rate rises substantially above the $1 \%$ claimed. High doses of oestrogen have been used, given for five days. During this time the patient takes as much oestrogen as in three years' cyclical use of a low-dose combined pill. Yet, despite its vast dose, this regimen has not been linked with thrombosis, though changes in clotting factors have been reported. ${ }^{4}$ The main problem is an incidence of ectopic pregnancy of about $10 \%$ in those in whom the method fails; that, combined with one report of pulmonary oedema, ${ }^{2}$ means that high-dose oestrogen regimens have fallen out of favour.

More recently Yuzpe and colleagues have devised an oestrogen-progestogen regimen in which the combination is taken in two doses 12 hours apart, ${ }^{56}$ with a total oestrogen content equivalent to about six low-dose combined pills. This regimen appears to be very safe; no complications have been reported. Yuzpe's method is also more acceptable than highdose oestrogens with respect to side effects. Nausea occurs in $50-60 \%$ of those treated and vomiting in $30 \%$, but the symptoms are short lived and rarely severe. ${ }^{6} 7$ Complaints of headache, dizziness, tenderness of the breasts, and withdrawal bleeding are fewer.

The possibility that administration of an oestrogenprogestogen combination just before implantation could be teratogenic (as stilboestrol can be during the first trimester of an established pregnancy ${ }^{2}$ ) has probably made clinicians overcautious. Though there may never be enough evidence to disprove an association, for practical purposes this potential risk can largely be ignored. ${ }^{8}$ Particular care should be taken, however, when counselling women with a moral or religious objection to abortion who say that they would continue with a pregnancy if the method failed, as $2.5 \%$ of all babies have some degree of congenital abnormality. ${ }^{2}$

The circumstances in which insertion of an intrauterine device is the preferred method include contraindications to the pill, the woman's choice of an intrauterine device for future contraception, and instances where hormones are less effective - such as presentation later than 72 hours after intercourse and when unprotected intercourse has taken place on more than one occasion. Surprisingly, the intrauterine device has a very low failure rate. No pregnancies have been reported in almost 700 postcoital insertions. ${ }^{2} 9$

The disadvantages of intrauterine devices must be fully explained to women who contemplate this method. Pain and abnormal bleeding are not uncommon problems, and the increased risk of pelvic inflammatory disease has serious implications: insertion of an intrauterine device should be avoided if at all possible in young nulliparae and in victims of rape.

The likelihood of conception at mid-cycle is at most $30 \% .{ }^{10}$ Interviews with women who request advice sometimes end with a decision that no action will be taken if the risk of pregnancy is judged to be negligible. Women who have had intercourse late in a regular menstrual cycle can often be reassured that they will not conceive. If there is any doubt about dates, however, treatment should not be withheld. Treatment may also seem justifiable simply because the woman is highly anxious.

Up to the present, hormone treatment has been prescribed by doctors only after counselling and examination. Some doctors are now advocating that a special "first-aid" pack of pills should be issued to users of sheaths in case these break" or as an "insurance policy" to those not currently in need of contraception. Even when contraindications to the pill have been excluded at the time of issue, there are still pitfalls in self-administered "morning-after" treatment. Of greater concern is the possibility that women will use borrowed pills when frequently either the dosage or the preparation would 